\title{
EXCITED HYDROGEN CHEMISTRY IN PROTOSTELLAR OUTFLOWS
}

\author{
J.M.C. RAWLINGS, J.E. DREW \\ Department of Physics \\ Nuclear Physics Laboratory \\ Keble Road, Oxford OX1 3RH, UK \\ M.J. BARLOW \\ Department of Physics and Astronomy \\ University College London \\ Gower street, London WC1E 6BT, UK
}

\begin{abstract}
Chemical models of protostellar and other outflows have been reassessed in the light of new chemical data. In particular, reactions involving excited hydrogen $(2 \mathrm{~s}, \mathrm{p})$ are shown to be important in hot, dense outflows. The $\mathrm{H}(\mathrm{n}=2)+\mathrm{H} \rightarrow \mathrm{H}_{2}+\mathrm{h} \nu$ reaction is much less of a contributor to the $\mathrm{H}_{2}$ formation rate than the recently measured $\mathrm{H}(\mathrm{n}=2)+\mathrm{H} \rightarrow \mathrm{H}_{2}^{+}+\mathrm{e}^{-}$reaction, providing conditions allow the $0.75 \mathrm{eV}$ endothermicity of this reaction to be overcome.
\end{abstract}

\section{Chemistry}

We report here for the first time the significance of the associative ionization reaction:

$$
H+H(n=2) \longrightarrow H_{2}^{+}+e^{-} .
$$

Cross-sections for this reaction have been measured by Urbain et al. (1991). Using these we calculate rate coefficients (in $\mathrm{cm}^{3} \mathrm{~s}^{-1}$ ) of: $1.7 \times 10^{-13}$ at $3000 \mathrm{~K}, 1.1 \times 10^{-12}$ at $5000 \mathrm{~K}$, and $6.0 \times 10^{-12}$ at $10000 \mathrm{~K}$. A good fit to the rate coefficient at $\mathrm{T} \gtrsim 2500 \mathrm{~K}$ is given by:

$$
k=3.5 \times 10^{-14}+3.1 \times 10^{-11} e^{-16355 / T} \mathrm{~cm}^{3} \mathrm{~s}^{-1}
$$

The reaction is endothermic by $0.75 \mathrm{eV}$ and the cross-section shows a secondary barrier of $\simeq 2 \mathrm{eV} . \mathrm{H}_{2}$ formation then occurs as a result of charge exchange:

$$
\mathrm{H}_{2}^{+}+\mathrm{H} \longrightarrow \mathrm{H}_{2}+\mathrm{H}^{+}
$$

We expect this path is particularly important in the chemistry of protostellar outflows. In all cases of astrophysical interest it is faster than the radiative association reaction proposed by Latter and Black (1991):

$$
H+H(n=2) \longrightarrow H_{2}+h \nu
$$

Furthermore, the negative-ion $\mathrm{H}_{2}$ formation route is ineffective in outflows from cool as well as hot stars because of photo-detachment of $\mathrm{H}^{-}$in the IR radiation field. In short, 
reaction (1) is a major contributor to the $\mathrm{H}_{2}$ formation rate in both (i) high-temperature, strongly irradiated regions where the $n=2$ level is well populated, (ii) cooler (3000 - 5000 $\mathrm{K}$ ), denser regions. In (ii), lower ionization and large departures from LTE (eg. at $3000 \mathrm{~K}$, $\left.\mathrm{H}(\mathrm{n}=2) / \mathrm{H}^{+}>2 \times 10^{-4}\right)$ are necessary for reaction (1) to be more important than direct radiative recombination of $\mathrm{H}^{+}$with $\mathrm{H}$.

\section{Models}

We have tested the importance of the excited hydrogen chemistry in four situations: T-Tauri winds, cool neutral outflows, BN winds and the ejecta of supernovae.

In the case of T-Tauri winds and cool neutral outflows we have adapted the models of Rawlings et al. 1988 and a model of Glassgold et al. 1989 to accommodate the new chemistry. In both cases the initial ionization was calculated by balancing recombination against collisional ionization. The results show that within a few stellar radii $\mathrm{H}_{2}$ is significantly enhanced on including reaction (1). However, at later times the temperature and density drop whilst, in the case of the T-Tauri wind, the ionization level remains quite high. At this stage radiative recombination of $\mathrm{H}^{+}$with $\mathrm{H}$ becomes relatively more efficient (see also Latter and Black's discussion of $\mathrm{H}(\mathrm{n}=2)$ chemistry in the early universe). Note these calculations do not include Ly $\alpha$ trapping and so set a lower limit to the efficiency of the $\mathrm{H}(\mathrm{n}=2)$ chemistry.

The winds associated with BN-type objects are hot, dense and fairly well ionized - the most promising conditions for the excited hydrogen reactions. In our model $R_{*}=4 \times 10^{11}$ cm, $\dot{M}=1.0 \times 10^{-6} \mathrm{M}_{\odot} \mathrm{yr}^{-1}, v_{\infty}=150 \mathrm{~km} \mathrm{~s}^{-1}$, and the underlying radiation field is a Kurucz model atmosphere for $\mathrm{T}_{\text {eff }}=25000 \mathrm{~K}, \log (\mathrm{g})=4.0$. We adopt a slowly accelerating velocity law and a temperature profile consistent with the wind models of Drew (1989). Selfshielding for $\mathrm{H}_{2}, \mathrm{CO}$ and the carbon continuum have been included. To begin with, we use a two-level $\mathrm{H}^{\circ}$ atom + continuum: transfer in Ly $\alpha$ is accounted for using escape probabilities based on the Sobolev approximation, and the effect of Balmer continuum opacity is included. For the initial conditions we assume that $\mathrm{H}(\mathrm{n}=1) / \mathrm{H}(\mathrm{n}=2)$ are in detailed balance and that photoionization of $\mathrm{H}(\mathrm{n}=2)$ balances case $\mathrm{B}$ recombination. Preliminary calculations confirm $\mathrm{H}_{2}$ formation via reaction (1) is very important in hot, partly ionized winds.

In the case of supernovae we have looked at both the "core" and the "mantle" of the ejecta. Despite the presence of a strong, very non-thermal high energy electron flux in the core region, NLTE models of the ejecta of SN1987a (eg. Schmutz et al. 1990) show the departure coefficients for $\mathrm{H}^{\circ} \mathrm{n}=1,2$ and 3 are less than 10. Hence, as the temperature is also low $(2-3000 \mathrm{~K})$, there is unlikely to be a significant enhancement to the chemistry in the core. In the "mantle" region, observations indicate that the $n=2$ level may be markedly overpopulated. However, even here, the importance of excited hydrogen chemistry in SN1987a appears to be marginal.

In some situations, the reaction: $H(n=3)+H \rightarrow H_{2}^{+}+e^{-}$may also be significant. It is exothermic by $1.14 \mathrm{eV}$ but no cross-sections are yet available.

\section{References}

Drew, J.E. 1989, Astrophys. J. Suppl., 71267

Glassgold, A.E., Mamon, G.A. \& Huggins, P.J. 1989, Astrophys. J., 336 L29

Latter, W.B. \& Black, J.H. 1991, Astrophys. J., 373161

Rawlings, J.M.C., Williams, D.A. \& Canto, J. 1988, Mon. Not. R. ast. Soc., 230695

Schmutz, W., et al. 1990, Astrophys. J., 355255

Urbain, X., Cornet, A., Brouillard, F. \& Giusti-Suzor, A. 1991, Phys. Rev. Lett., 661685 